\title{
Calidad de la rotación de pediatría comunitaria, desde la percepción del estudiante
}

\author{
Student's perception on community pediatric rotation quality
}

\author{
Luis Américo Reátegui Guzmán, Manuel Hernán Izaguirre Sotomayor² \\ ' Profesor Asociado, Departamento de Pediatría, Facultad de Medicina San Fernando, Universidad Nacional Mayor de San Marcos, Lima, Perú. \\ ${ }_{2}^{2}$ Profesor Principal, Departamento de Pediatría, Facultad de Medicina San Fernando, Universidad Nacional Mayor de San Marcos, Lima, Perú.
}

\begin{abstract}
Resumen
Introducción: Las investigaciones publicadas sobre la calidad de servicio de la educación superior universitaria se han concentrado en el contenido del curso y su entrega y no en la calidad del servicio. El instrumento utilizado para la medición de la calidad ha sido el modelo ServQual. Objetivos: Evaluar la calidad de servicio educativo en la rotación de Pediatría Comunitaria desde la perspectiva del estudiante. Diseño: Estudio transversal, descriptivo, de diseño cuantitativo. Lugar: Departamento de Pediatría, Facultad de Medicina San Fernando, UNMSM. Participantes: Alumnos de la rotación de Pediatría Comunitaria. Intervenciones: Aplicación de la encuesta ServQual antes y después de la rotación. Resultados: La satisfacción global para todas las dimensiones fue de satisfacción y el nivel insatisfacción severa fue mínimo. En las dimensiones clases, profesores, instalaciones, personal administrativo, información, comunicación con la universidad y entorno institucional se observó que la satisfacción media fue de insatisfacción con la importancia que se le da al servicio educativo. En las otras dimensiones fue de satisfacción. Las dimensiones instalaciones, vida social, personal administrativo, comunicación con la universidad, situación financiera y entorno institucional presentaron niveles bajos de insatisfacción severa. Conclusiones: La satisfacción global, la satisfacción media, la permanencia, la elección de la rotación, el funcionamiento y la salud durante la rotación de Pediatria Comunitaria para la mayoría de los estudiantes fueron de satisfacción, y los atributos a los cuales los alumnos les dieron mayor importancia fueron calidad de las clases y capacidad de los profesores por la enseñanza.
\end{abstract}

Palabras clave: Calidad de servicio, dimensiones de la calidad, ServQual.

\section{Abstract}

Introduction: Published research on university education quality of service has focused on course content and submission and not on the quality of service. The instrument used for measuring quality is the SERVQUAL model. Objectives: To determine the quality of education in Community Pediatrics rotation from the student's perspective. Design: Cross-sectional, descriptive, quantitative study. Location: Department of Pediatrics, San Fernando School of Medicine, Universidad Nacional Mayor de San Marcos. Participants: University Pediatrics rotation students. Interventions: SERVQUAL survey application before and after rotation. Results: Overall satisfaction for all dimensions was found and severe unsatisfaction was minimal. In size classes, teachers, facilities, staff, information, communication with the university and institutional environment unsatisfaction was median with importance given to educational service. Satisfaction was encountered in the other dimensions. Dimensions facilities, social life, administrative personal, communication with the university, financial and institutional environment showed low levels of severe unsatisfaction. Conclusions: Most students had overall satisfaction, median satisfaction, with satisfaction in permanency, rotation, performance and health during rotation of Community Pediatrics; more important attributes given by the pupils were quality of classes and teachers' capacity for teaching.

Key words: Quality of service, quality dimensions, ServQual.

An Fac med. 2013;74(2):117-21

\section{INTRODUCCIÓN}

El objetivo de la presente investigación fue evaluar la calidad de servicio educativo en la rotación de Pediatría Comunitaria en el 6 o año de estudios de medicina de la Facultad de Medicina de la Universidad Nacional Mayor de San Marcos, desde la perspectiva del estudiante, que contribuya a la comprensión de las percepciones de los estudiantes de la calidad del servicio en el sector de la educación superior. Por lo tanto, la presente investigación intenta poner a disposición de las autoridades universitarias una herramienta que les permita medir y gestionar la calidad del servicio percibida por sus estudiantes.

En algunos países se ha implementado sistemas de gestión de la calidad bajo la norma ISO 9001: $2000^{(1)}$, que exige el cumplimiento de una serie de criterios, incluyendo el monitoreo de la satisfacción del cliente, la evaluación de la información relativa a las percepciones del estudiante y al cumplimiento de las demandas del estudiante (ISO 9001:2000), donde la percepción de la calidad del servicio educativo por parte del estudiante es un elemento más de sus estándares exigidos.

Las universidades, en su afán por ser competitivos en el sector de la educación superior, están siendo obligadas a 
considerar la calidad percibida de los servicios educativos prestados desde la perspectiva de los estudiantes. Más aún, cuando el foco principal fue la calidad del producto académico y no la calidad del servicio percibido de la educación superior ${ }^{(2-4)}$.

Ahora bien, medir la calidad de un bien manufacturado es muy diferente a medir la calidad de los servicios educativos que las universidades brindan a los estudiantes, toda vez que son intangibles, además de ser influenciada por las funciones de servicios múltiples que contribuyen a la percepción de la calidad en general, y no pueden ser completamente inspeccionados antes de ser consumidos ${ }^{(5)}$.

Por otro lado, al ser la calidad del servicio un concepto multifacético y no haber un consenso claro en la literatura sobre el número de dimensiones y atributos, así como sobre su interrelación, según Hill ${ }^{(6)}$ deberían incluirse la centralidad del cliente, la relación entre sus expectativas y percepciones de la prestación del servicio, y la importancia que los clientes atribuyen a las diferentes dimensiones y atributos del servicio.

Las investigaciones publicadas sobre la calidad de servicio de la educación superior universitaria se han concentrado en el contenido del curso y su entrega. Pero, debido a la alta competitividad del sector en el contexto de un mundo globalizado ha obligado a las universidades a enfocarse en la calidad de los servicios que ofrecen ${ }^{(3)}$.

Finalmente, algunos investigadores han tratado de adaptar o mejorar el modelo SERVQUAL ampliamente reconocido en el ámbito de la educación superior universitaria, con diferentes niveles de éxito ${ }^{(7-10)}$.

Por último, Torres E. \& Araya L. ${ }^{(11)}$ construyeron una escala de medición de la calidad del servicio de las universidades contextualizadas a la realidad chilena, como herramienta que les permitía medir y gestionar la calidad percibida del servicio. Mancebón, Martínez \& Pérez ${ }^{(12)}$ analizaron las percepciones que tuvieron los estudiantes sobre distintos atributos de la calidad de la enseñanza que recibieron en los centros de educación secundaria españoles, encontrando diferencias estadísticamente significativas en la percepción de la calidad de los centros públicos y privados. Algunos investigadores (13) precisan haber desarrollado, evaluado y aplicado una escala de medida de la calidad de servicio en el ámbito universitario a partir de las percepciones de estudiantes, demostrando resultados satisfactorios tanto en lo que se refiere a la fiabilidad como a la validez. Así mismo, se identificó cinco dimensiones distintas, comprobándose que cada una de ellas tenía efecto positivo y significativo sobre la calidad global de la enseñanza. Otras investigaciones (14) abordaron el estudio de la medición de la calidad del servicio universitario en la Universidad de Málaga, utilizando el modelo ServQual. Investigadores venezolanos ${ }^{(15)}$ publicaron una experiencia en la educación superior de su país basándose en el modelo ServQual, determinando una alta consistencia interna y pertinencia de la aplicación del análisis de factores, dándole validez y fiabilidad. Caetano ${ }^{(16)}$ investigó los factores de calidad del servicio aplicados a la enseñanza superior de marketing en instituciones públicas y privadas adaptando el modelo ServQual, para el ámbito en el que se desarrolla la enseñanza superior de marketing. Moreno ${ }^{(17)} \mathrm{di}$ señó el SoftPerf basándose en el modelo ServPerf ${ }^{(18)}$, para la medición de la calidad percibida, el mejoramiento y control de los servicios.

\section{MÉTODOS}

El estudio fue transversal, descriptivo, de diseño cuantitativo. La muestra estuvo conformada por 82 estudiantes de la rotación de Pediatría Comunitaria, entre los meses de enero a diciembre de 2011. Las unidades de análisis estuvieron conformadas por los estudiantes de la rotación de Pediatría Comunitaria.

Al no haberse utilizado métodos invasivos que pusieran en peligro la salud física y mental de los estudiantes, no existieron problemas éticos en este aspecto. Sin embargo, se respetó los principios éticos aceptados por la sociedad y los estipulados por el Comité de Ética de la Universidad, así como la propiedad intelectual. La identidad de los estudiantes fue reemplazada por códigos, al procesar y comunicar los resultados.

El instrumento que se utilizó fue el SERVQUAL modificado, el cual ha sido ampliamente empleado para la evaluación de la calidad de los servicios en el Perú y el mundo. Las dimensiones de la calidad consideradas para el estudio fueron: clases, profesores, instalaciones, vida social, personal administrativo, información, comunicación con la universidad, situación financiera y entorno institucional.

La interpretación de las diferencias entre expectativas y percepciones (grado de satisfacción de los usuarios) se realizó considerando los siguientes parámetros:

- Satisfacción amplia $(-4$ a -1)

- Satisfacción (0)

- Insatisfacción leve/moderada (1 a 2)

- Insatisfacción severa (3 a 4)

Los parámetros empleados estuvieron basados en los establecidos por Parasuraman y colaboradores y posteriormente adaptados por diversos autores para la encuesta SERVQUAL modificada.

La técnica utilizada fue la encuesta directa cara a cara. El instrumento utilizado para el recojo de los datos fue el SERVQUAL modificado, que fue aplicado por un encuestador ajeno al servicio y capacitado en el manejo y procedimiento de su aplicación. El instrumento SERVQUAL fue aplicado en dos momentos; la primera parte de la encuesta -datos generales y preguntas de expectativas- fue aplicada antes de que el usuario recibiera los servicios educativos y la segunda parte después de recibir el servicio.

Los datos obtenidos fueron ingresados en Microsoft Office Excel 2000 y 
tabulados mediante software SPSS versión 15

Finalmente, se realizó el análisis descriptivo de todas las variables, a través de la determinación de proporciones de las variables sociodemográficas y medias de satisfacción de expectativas y percepciones.

En la ejecución de la investigación no se presentaron limitaciones importantes al desarrollo del estudio, salvo las inasistencias de los alumnos a una de las dos intervenciones, lo que determinó la exclusión del estudio de varios estudiantes.

\section{RESULTADOS}

Los datos sociodemográficos revelaron que los alumnos de Pediatría Comunitaria, mayoritariamente, tuvieron una edad entre 22 a 25 años, fueron varones y procedían de Lima.

El promedio de los puntajes de la percepción de los alumnos acerca del servicio educativo fue mayor al promedio de los puntajes de la expectativa de los alumnos en las dimensiones clases, profesores, instalaciones, personal administrativo, información, comunicación con la universidad, situación financiera y entorno institucional. Contrariamente, en la dimensión vida social el promedio de las expectativas fue mayor al promedio de las percepciones.

El nivel de satisfacción global de los alumnos respecto al servicio educativo para la dimensión clases, profesores, instalaciones y vida social fue de $58 \%$, $60 \%$, $68 \%$ y $51 \%$, respectivamente. En cuanto a la dimensión personal administrativo, información, comunicación con la universidad, situación financiera y entorno institucional fue de $62 \%$, $54,7 \%, 53 \%, 40 \%$ y $49 \%$, respectivamente (figura 1).

Con relación a la satisfacción global, el $62 \%$ de alumnos se encontró satisfecho, mientras que el $32 \%$ restante presentó una insatisfacción.

Respecto a la importancia que se le dio al servicio educativo en la dimen- sión clases, profesores, instalaciones, personal administrativo, información y entorno institucional, fue desfavorable, contrariamente a las dimensiones vida social y situación financiera que fueron favorables.

En cuanto al nivel de satisfacción global de los alumnos respecto a la importancia dada al servicio educativo, se pudo constatar, en cuanto a la dimensión clases, profesores, instalaciones, vida social, personal administrativo, información, comunicación con la universidad, situación financiera y entorno institucional, resultados de $46 \%, 43 \%$, $42 \%, 46 \%, 43 \%, 41 \%, 40 \%, 39 \%, 40 \%$, respectivamente.

En cuanto al servicio educativo en Pediatría Comunitaria, 46\% de alumnos se encontraba satisfecho y el $32 \%$ restante presentó insatisfacción.

La investigación también reveló que $45 \%$ de los alumnos se encontraba satisfecho de su permanencia en la rotación de Pediatría Comunitaria. Mientras que 37\% de los alumnos pre-

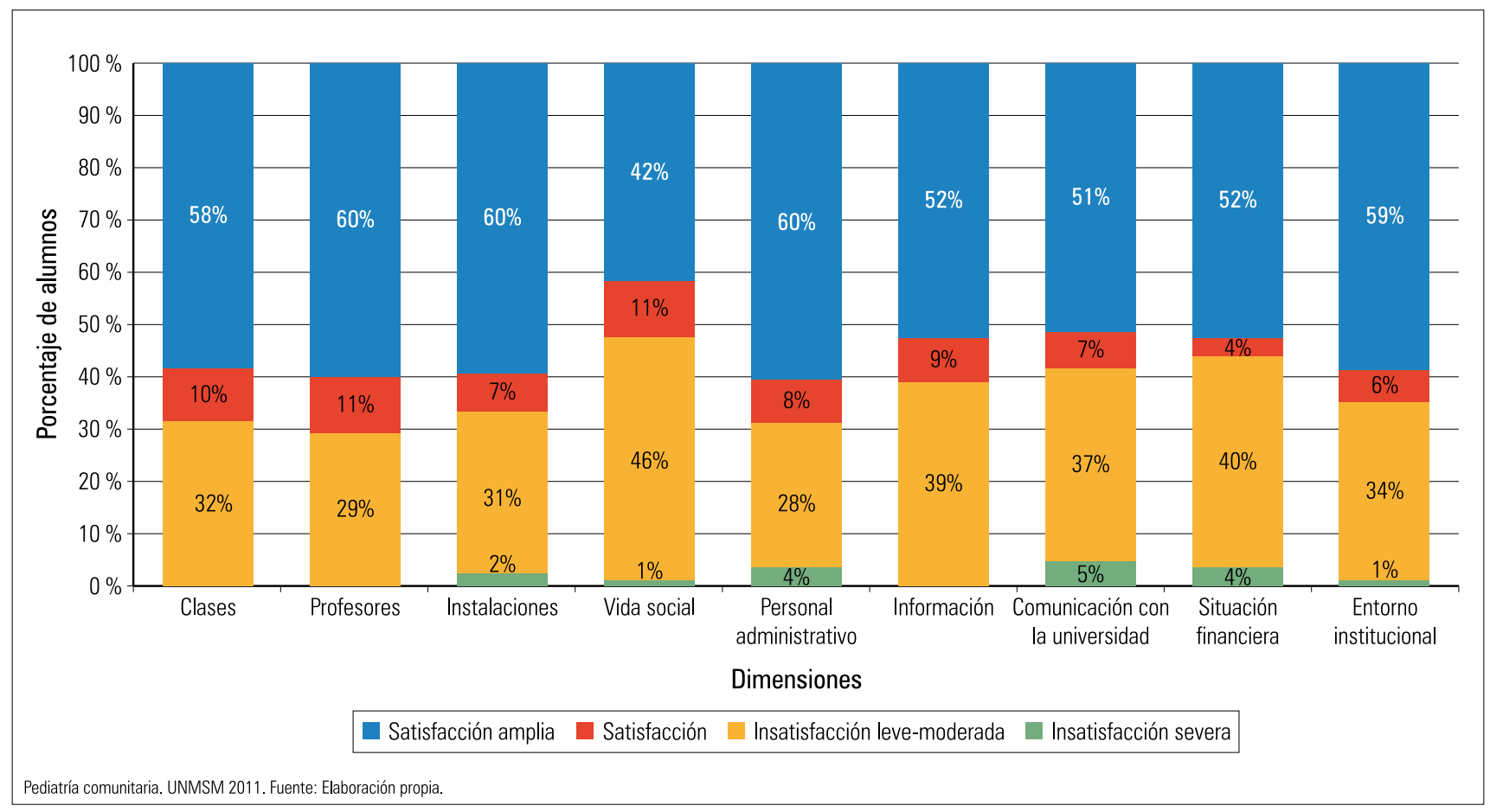

Figura 1. Nivel de satisfacción global por dimensiones de la calidad educativa. 
sentó insatisfacción. En cuanto al nivel de satisfacción de los alumnos con su elección de la rotación de Pediatría Comunitaria, se evidenció que $80 \%$ de los alumnos se encontró satisfecho.

Con relación al funcionamiento de la rotación de Pediatría Comunitaria, se pudo comprobar que $78 \%$ de los alumnos se encontraba satisfecho. En cuanto a su estado de salud durante la rotación de Pediatría Comunitaria, 65\% de los alumnos se encontró satisfecho.

Respecto a la permanencia en el país, el 50\% tenía la expectativa de quedarse en el país y $72 \%$ deseaba quedarse en el país. En referencia a los motivos por los que los alumnos querían dejar el país al finalizar sus estudios, la encuesta reveló que estos fueron motivos académicos, privados, la carrera profesional y mejores oportunidades. Contrariamente, los motivos por los cuales deseaban quedarse en el país fueron ayudar a mejorar la salud del país, motivos académicos y retribuir al país los estudios financiados por el Estado. Por otro lado, el nivel de satisfacción de los alumnos acerca de su situación financiera durante su permanencia en la universidad el estudio reveló que $77 \%$ de los alumnos se encontraba satisfecho.

Con respecto a los atributos a los cuales los alumnos les dieron mayor importancia fueron calidad de las clases y capacidad de los profesores por la enseñanza, mientras que los atributos claridad de dónde conseguir información e información sobre Pediatría Comunitaria en Internet, tendieron a tener menor relevancia para los alumnos.

\section{DISCUSIÓN}

Las expectativas de los alumnos respecto al servicio educativo que brinda la institución, en casi todas sus dimensiones, fueron superadas por la institución. Lo cual se refleja en los promedios de satisfacción obtenidos, los cuales fueron favorables, porque en la mayoría de las dimensiones tendieron a estar en la escala de parámetros con valores negativos que indican satisfacción. Solo el promedio que tendía a ser positivo fue el obtenido en la dimensión vida social.

Como se apreció en el resultado, los promedios de satisfacción por el servicio educativo fueron favorables a la institución, lo cual debería estar en relación a un resultado parecido respecto a la satisfacción de la importancia que se le da al servicio educativo brindado; pero, no fue así; según los promedios obtenidos, los alumnos tendieron a estar insatisfechos. Sin embargo, la satisfacción global, la satisfacción global por dimensiones y la satisfacción media de la calidad del servicio educativo para la mayoría de los estudiantes fueron de satisfacción. Un porcentaje representativo de estudiantes ( $40 \%$ a más) estuvo insatisfecho en las dimensiones clases, profesores, instalaciones, vida social, personal administrativo, comunicación con la universidad, entorno institucional.

Por tanto, es posible cuestionar el resultado encontrado, debido a posibles causas que pudieron intervenir en la percepción de los alumnos. Por ejemplo, amerita preguntarse si existe una buena difusión a la comunidad universitaria de las acciones que la institución realiza con el fin de brindar un servicio educativo de calidad, aspecto que en el presente trabajo no se tomó en cuenta.

Asimismo, es posible ver también que la diferencia entre los promedios de expectativa y percepción fue mínima. Recordemos que el resultado respecto al promedio de satisfacción por el servicio educativo en general $(-0,18)$ fue favorable a la institución, evidenciándose satisfacción media en las dimensiones clases, profesores, instalaciones, personal administrativo, información, comunicación con la universidad, situación financiera y entorno institucional, pues fue de satisfacción para la mayoría de los estudiantes.

Por lo tanto, no guarda relación con el resultado obtenido respecto al promedio de satisfacción por la importancia que se le da al servicio educativo en general $(0,03)$, debido a que, si bien se aproxima a cero, tiende a ser positivo, lo cual significa un nivel mínimo de insatisfacción, no siendo favorable a la institución. Por lo tanto, los porcentajes de estudiantes insatisfechos obligan a la mejora constante del servicio educativo que se brinda.

Este resultado amerita indagar respecto a posibles causas que pudieron intervenir en la percepción de los alumnos. Por ejemplo, preguntarse si la difusión a la comunidad universitaria, de las acciones que la institución realiza con el fin de brindar un servicio educativo de calidad, es la adecuada. Sin embargo, es preciso remarcar que la satisfacción global de la importancia dada al servicio educativo fue de satisfacción para la mayoría de los estudiantes $(56 \%)$, a pesar que un porcentaje representativo de estudiantes (42\%) estuvo insatisfecho.

La percepción de los alumnos respecto a la importancia que la institución le da al servicio educativo puede estar influenciada por su ignorancia respecto a las acciones que realiza la institución internamente por mejorar el servicio educativo (por ejemplo, capacitaciones al personal docente y administrativo) y que no son difundidas.

En general, se puede ver que en todas las dimensiones la mayoría de alumnos (superan el 50\%) se encuentra satisfecho y/o satisfecho ampliamente, con la importancia que se le da al servicio educativo. Sin embargo, la contradicción entre la percepción no satisfactoria de los estudiantes respecto a la importancia que se le da al servicio educativo y la satisfacción por la calidad del servicio educativo brindado, obliga a investigar sobre las posibles causas que la originan.

Es importante mencionar también que, en las dimensiones clases, instalaciones y entorno institucional, los porcentajes de alumnos en el nivel insatisfacción leve-moderada superaron el $40 \%$, siendo necesario tomar medidas con el fin de reducir estos porcentajes. Por otra parte, se observa que los porcentajes en el nivel de insatisfacción 
severa fueron mínimos, no superaron el $4 \%$.

Si bien, el porcentaje de alumnos satisfechos supera el 50\%, se observa también que es necesario reducir el porcentaje de alumnos insatisfechos, siendo necesario tomar medidas con el fin de reducir estos porcentajes.

Respecto a las expectativas, se puede ver en general que el porcentaje de alumnos que sí deseó quedarse en el país al finalizar sus estudios fue mayor al porcentaje de alumnos que deseó dejar el país. Por tanto, la mayoría (más del 70\%) de los alumnos encuestados se encontró satisfecha y/o ampliamente satisfecha con su permanencia en el país después de finalizar sus estudios. Así mismo, la mayoría de estudiantes se encontró satisfecha con la elección de la rotación, su funcionamiento y su salud, durante la rotación de Pediatría Comunitaria.

Igualmente, la mayoría (más de 70\%) de los alumnos encuestados se encontró satisfecha y/o ampliamente satisfecha con su situación financiera durante su permanencia en la universidad. Finalmente, para los alumnos los atributos que tuvieron mayor importancia fueron calidad de las clases y capacidad de los profesores por la enseñanza.

En conclusión, la satisfacción global y la satisfacción media para la mayoría de los estudiantes fueron de satisfacción, mientras que la satisfacción media en las dimensiones clases, profesores, instalaciones, personal administrativo, información, comunicación con la universidad, situación financiera y entorno institucional fue de satisfacción para la mayoría de los estudiantes. Sin embargo, los porcentajes de estudiantes insatisfechos obligan a la mejora constante del servicio educativo que se brinda. Finalmente, los atributos a los cuales los alumnos les dieron mayor importancia fueron calidad de las clases y capacidad de los profesores por la enseñanza.

\section{REFERENCIAS BIBLIOGRÁFICAS}

1. ISO 9001:2008 Quality management systems requirements, Standards Australia/Standards New Zealand. Obtenida en: https://infostore.saiglobal. com/store/PreviewDoc.aspx?...03-06-11.

2. O'Neill M, Wright C, Fitz, F. Quality evaluation in on-line service environments: an application of the importance-performance measurement technique. Managing Service Quality. 2001;11(6):402-17.

3. O'Neill M. The role of perception in disconfirmation models of service quality. Measuring Business Excellence. 2000;4(2):46-59.

4. O'Neill M. The influence of time on student perceptions of service quality. The need for longitudinal measures. J Educational Administration. 2003;41(3):310-24.

5. Didomenico E, Bonnici J. Assessing service quality within the educational environment. Education. 1996;116(3):353-59.

6. Hill F. Managing service quality in higher education: The role of the student as primary consumer. Quality Assurance in Education. 1995;3(3):10-21.

7. Anderson E. High tech v. high touch: a case study of TQM implementation in higher education. Managing Service Quality. 1995;5(2):48-56.

8. Anderson E, Zwelling L. Measuring service quality at the University of Texas M.D. Anderson Cancer Center. Internat J Health Care Qual Assur. 1996;9(7):922.

9. Soutar G, McNeil M. Measuring service quality in a tertiary institution. J Educational Administration. 1996;34(1):72-82.

10. Pariseau S, McDaniel J. Assessing service quality in schools of business. Internat J Qual Reliabil Management. 1997;14(3):204-18.

11. Torres E, Araya L. Construcción de una escala para medir la calidad del servicio de las universidades: Una aplicación al contexto chileno. Rev Ciencias Sociales. 2010;16(1). Recuperado de http://www. revistas.luz.edu.ve
12. Mancebón-Torrubia M, Martínez-Caraballo N, PérezXiménez D. Un análisis de la calidad percibida por los estudiantes en los centros públicos y privados de la enseñanza secundaria. Obtenida de http:// works.bepress.com 25-06-11.

13. Capelleras J, Veciana M. Calidad de servicio en la enseñanza universitaria: desarrollo y validación de una escala de medida. Universidad Autónoma de Barcelona 2001. Obtenido de http://www.recercat. net 16-06-11.

14. Garcia-Mestanza J, Diaz-Muñoz R. Comparativa entre distintos sistemas de medición de calidad de servicio. EsicMarket. 2008;130:57-97. Obtenido de http://www.esic.es 10-06-11.

15. Maneiro N, Mejías A, Romero M, Zerpa J. Evaluación de la calidad de los servicios, una experiencia en la educación superior venezolana. EDUCERE 2008; pp. 797 - 804. Investigación arbitrada. Universidad de Carabobo. Valencia, Venezuela. Obtenido de http://redalyc.uaemex.mx 10-06-11.

16. Caetano G. Marketing en los servicios de educación: modelos de percepción de calidad. Memoria para optar el grado de Doctor. Universidad Complutense de Madrid 2003. Obtenido de www.ucm.es 16-0611 .

17. Moreno M. Procedimiento para la medición de la calidad percibida, mejoramiento y control de los servicios de DESOFT S.A. En: Montaña J, Ramírez E, Ramírez H. Evaluación de la calidad de los servicios públicos domiciliarios. Rev Colombiana Marketing. 2010. Recuperado de http://semanatecnologica. fordes.co.cu 16-06-11.

18. Cronin J, Taylor S. Measuring service quality: a reexamination and extension. J Marketing. 1992;56:55-68.

Artículo recibido el 10 de julio de 2012 y aceptado para publicación el 28 de enero de 2013.

Fuentes de financiamiento: Propias

Conflicto de intereses:

Los autores expresan no tener algún conflicto de interés.

Correspondencia:

Dr. Luis Reátegui Guzmán

Facultad de Medicina Humana

Correo-e: reategui.asesores.salud@peru.com 